\title{
Interventions to Increase Referrals to a New Pediatric $\square$ Palliative Care Service: A QI project
}

\author{
Kathleen Murphy and Ratna B Basak* \\ Department of Pediatrics, Stony Brook University Hospital, USA
}

Received: 㘹 July 01, 2018; Published: 監August 09, 2018

*Corresponding author: Ratna Basak, Department of Pediatrics, Stony Brook University Hospital, New York

\section{Short Communication}

Pediatric palliative care is still a young, but rapidly growing field world wide. There have been a number of children's hospitals creating palliative care services over the past two decades. Both the American Academy of Pediatrics and Institute of Medicine have focused attention on increasing the care access for children with life-threatening or life-limiting medical conditions. Data has shown the beneficial effects of inpatient palliative care services, not only on assisting control of physical symptoms like pain but also to ameliorate the suffering and maintain dignity that is appropriate for the culture, religion and upbringing of the family. The service has shown to improve patient satisfaction and decrease the length of stay. Various studies have projected newly formed pediatric palliative care teams initial struggles, periods of quiescence with variable levels of growth and success over subsequent years.

\section{Objectives}

To track the effect of various interventions on the number of consult referrals to a new pediatric palliative care service.

\section{Methods}

All primary and repeat referrals to pediatric palliative care were recorded over the first year after the service was created. Direct interventions with staff included division meetings with pediatric pulmonology, NICU and PICU to discuss the role of palliative care. Huddles were done with the nursing staff from NICU, PICU, Pediatric Oncology and the Pediatric floor and meetings were conducted with the social workers covering these units. Other interventions included grand rounds, website creation, and lectures on palliative care to medical students.

\section{Primary Data and Goal Description}

Initial interventions occurred a few months after the palliative care service officially started and consults immediately started to occur. When new interventions were added from December to March, consult numbers increased to a new level, though they did not seem to continue to increase month to month (Figure1). The increase in consults was most likely an effect of the reminder that the new palliative care service was in place and available. Future planned interventions within the next 1 year (April 17-April 18) include regularly scheduled meeting with social workers, a colloquium lecture and a brochure. Other division meetings are also being planned, including hematology/oncology. Possible interventions in the future could also include screening criteria to trigger automatic palliative care consults for patients with certain chronic or life-threatening conditions, and a system to ensure repeat consults when a patient already known to the service is readmitted to the hospital, resident elective rotations, informal lunch discussions periodically.

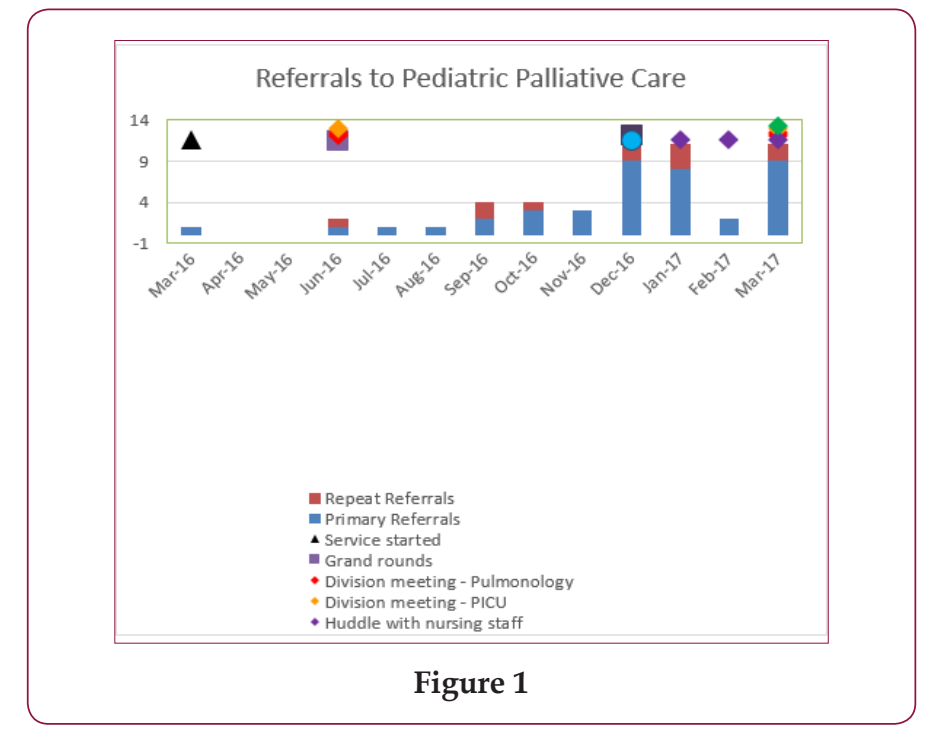

\section{Conclusion}

We anticipate that with this planned QI project, the awareness of importance of Pediatric Palliative Care Service will increase and more children with complex medical and life-threatening illness will be referred to the service, with an increase of $50 \%$ from baseline. 


\section{ISSN: 2574-1241}

DOI: 10.26717/BJSTR.2018.07.001555

Ratna Basak. Biomed J Sci \& Tech Res

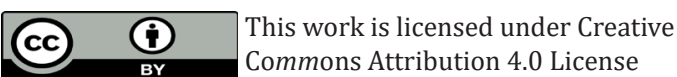

Submission Link: https://biomedres.us/submit-manuscript.php

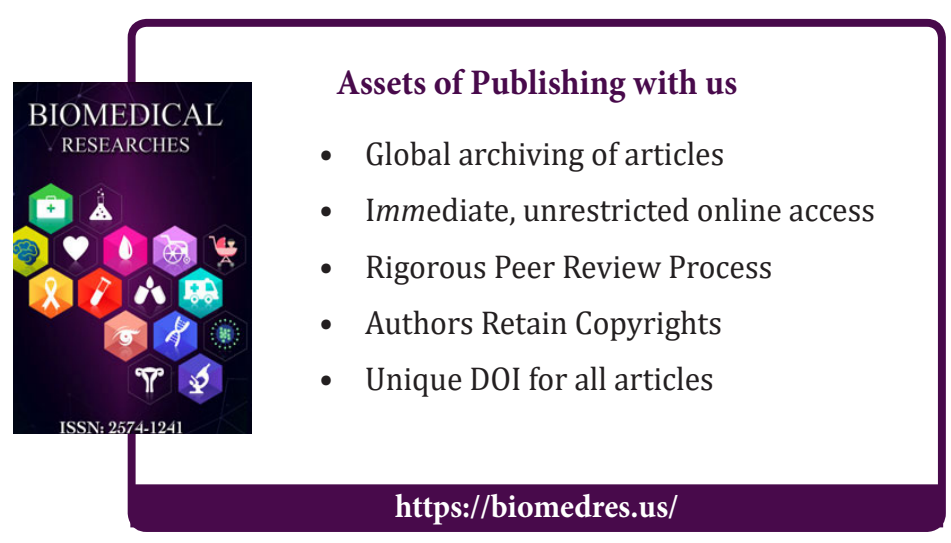

